\title{
Automated Incident Detection Using Real-Time Floating Car Data
}

\author{
Maarten Houbraken, ${ }^{1,2}$ Steven Logghe, ${ }^{2}$ Marco Schreuder, ${ }^{3}$ Pieter Audenaert, \\ Didier Colle, ${ }^{1}$ and Mario Pickavet ${ }^{1}$ \\ ${ }^{1}$ Department of Information Technology, Ghent University, Gent, Belgium \\ ${ }^{2}$ Be-Mobile, Melle, Belgium \\ ${ }^{3}$ Rijkswaterstaat, Rijswijk, Netherlands \\ Correspondence should be addressed to Maarten Houbraken; maarten.houbraken@ugent.be
}

Received 5 July 2017; Revised 9 November 2017; Accepted 19 November 2017; Published 11 December 2017

Academic Editor: Ludovic Leclercq

Copyright (C) 2017 Maarten Houbraken et al. This is an open access article distributed under the Creative Commons Attribution License, which permits unrestricted use, distribution, and reproduction in any medium, provided the original work is properly cited.

\begin{abstract}
The aim of this paper is to demonstrate the feasibility of a live Automated Incident Detection (AID) system using only Floating Car Data (FCD) in one of the first large-scale FCD AID field trials. AID systems detect traffic events and alert upcoming drivers to improve traffic safety without human monitoring. These automated systems traditionally rely on traffic monitoring sensors embedded in the road. FCD allows for finer spatial granularity of traffic monitoring. However, low penetration rates of FCD probe vehicles and the data latency have historically hindered FCD AID deployment. We use a live country-wide FCD system monitoring an estimated 5.93\% of all vehicles. An FCD AID system is presented and compared to the installed AID system (using loop sensor data) on 2 different highways in Netherlands. Our results show the FCD AID can adequately monitor changing traffic conditions and follow the AID benchmark. The presented FCD AID is integrated with the road operator systems as part of an innovation project, making this, to the best of our knowledge, the first full chain technical feasibility trial of an FCD-only AID system. Additionally, FCD allows for AID on roads without installed sensors, allowing road safety improvements at low cost.
\end{abstract}

\section{Introduction}

Traditionally, traffic monitoring has largely relied on dedicated roadside equipment. Sensor equipment such as inductive loop detectors, wireless signal receivers, or cameras were installed on or embedded in the road surface to detect all individual vehicles passing the specific location. This yields a trove of traffic data to be used in traffic state estimation, travel time measurements, and traffic management applications. While loops only monitor a single location, vehicle reidentification techniques can be applied to obtain vehicle trajectory information, allowing for various application, for example, estimating travel times $[1,2]$, highway monitoring [3], and incident detection [4-6]. A complete system of connected measurement locations can be installed, allowing road operators to monitor traffic. However, total system costs (see [7] for a selection of installed ITS systems and costs) scale linearly with the covered area size. While the actual loop hardware is relatively inexpensive, the installation costs (e.g., highways closures, road surface cuts, and power supply) and maintenance (e.g., monitoring loop status, closing individual lanes, and repairing broken network cables) greatly impact the total cost during the full system lifespan. While loop data quality has improved over the past years, loop systems are still prone to malfunction. In [8], diagnostics showed $31 \%$ of loop sensors in a Chinese city to be defect and $25 \%$ of the remaining sensors reporting errors $>20 \%$ compared to visual counts. This indicates the need for better data.

With mobile devices becoming prevalent in the past few years, an invaluable source of traffic data has become available termed as Floating Car Data (FCD). The FCD itself consists of measurements coming from individually tracked probe vehicles reporting their location and speed. The data generally consists of timestamped positional information, along with the instantaneous vehicle speed. As vehicles are tracked on their route, the data is, in contrast to loop detector data, 
inherently spatially distributed. This results in a completely different FCD system cost model. While the hardware cost of an FCD system is limited to a few central servers, getting (or buying) live data from probe vehicles becomes the main issue. However, the data can be sourced from other applications, for example, existing track and trace platforms or mandatory tolling systems, thus sharing the costs, and additional (profitable) services using the same data can be deployed (e.g., insurance). As more and more cars are getting connected, the amount of data will only increase, allowing for largescale FCD AID deployment at a fraction of loop-based system costs.

Additionally, the FCD differs from the loop detector data in terms of total vehicle coverage, as it only monitors a limited sample of the total traffic. Comparing FCD with loop detector data is focused on in [9], noting statistical differences in calculated travel times which need to be accounted for. The sampling rate and penetration coverage are studied in [10], showing the suitability of current FCD techniques in data fusion.

While most research is focused on traffic state and travel time estimation, FCD can be used for a wide range of applications like traffic policy evaluation [11] or road network mapping/generation [12]. Another closely related application is Automated Incident Detection (AID) in which traffic is monitored using live loop detectors, FCD, and other sources to obtain a view on traffic conditions. They try to avoid congestion and collisions by activating overhead speed restriction signs in order to homogenize traffic and alert drivers for upcoming traffic events. This improves traffic by improving congestion recovery and safety by avoiding end-of-queue collisions [13]. Combining even small amounts of FCD with existing loop-based systems greatly increases performance [14]. Various data fusion algorithms have also been developed, focusing on combining FCD with roadside detector data. These algorithms combine the information in both sources to better estimate traffic variables [15-17]. However, this paper focuses on pure FCD data; a more comprehensive review of data fusion algorithms can be found in [18].

While FCD definitely can contribute to existing loopbased systems, it could also be directly used to feed incident detection algorithms. In [19], 2 AID algorithms (1 using probe travel times and 1 using shockwave theory) are presented using a simulated AIMSUM FCD dataset. Simulated FCD studies typically model the underlying network and fit the model to target traffic volumes, supply-demand matrices, or loop detector measurements. The calibrated model is then sampled to obtain FCD to be used in experiments. This approach allows varying the penetration rates and testing FCD algorithms for performance. A traffic state classification AID is presented in [20] using simulated FCD generated by SUMO, calibrated using an unspecified set of real FCD traces near Pisa, Italy. They note the lack of real FCD as the main reason for simulation. Traffic state estimation and AID were tackled together in [21] using a CORSIM simulation in a hybrid state estimation problem. However, all these results are still dependent on model parameters and assumptions.

Studies using real-time FCD, captured from actual vehicles driving on the studied routes, do not have model assumptions but large FCD systems are still being rolled out. However, several field systems have been successfully deployed. The Mobile Century project [22] gives the results for 100 dedicated vehicles driving loops on a highway, giving an estimated 2-3\% needed penetration rate. In Stockholm, a fleet of 1500 taxis has been used to estimate route travel times and travel time distributions in $[23,24]$, focusing on eliminating inherent biases. Several comparable taxi FCD systems have also been deployed in Berlin (200 taxis), Vienna (400), and Nuremburg (500) in [25]. A real-time AID system using an underlying state classification for traffic on an hourly basis was reported in [26], although no specific information is given on the actual real-time detection time or FCD coverage. However, most of these systems are limited to cities.

To eliminate the model influences and city-limited FCD properties mentioned above, this paper presents the results of an FCD AID algorithm working solely on FCD from a country-wide system covering around $6 \%$ of all traffic with anonymized data being collected from a free transportation app. Data from this system was used to feed a real-time FCD AID algorithm. To further prove the operational feasibility, the output of the algorithm was transmitted real-time to Dutch roadside operators and integrated in their monitoring setup, contrary to most literature on FCD limited to local test setups. The main focus of this study is to examine the current feasibility of FCD systems to provide live signage for large area AID systems, taking into account technical issues and coupling back to existing infrastructure. By establishing an entire operational FCD AID chain, our study provides unique and quantifiable results on current day FCD AID feasibility.

The rest of this paper is structured as follows. Section 2 gives details on the installed loop AID algorithm and benchmark while Section 3 shows the FCD modifications to the existing algorithm. Section 4 elaborates on the evaluation methodology used in this paper, consisting of a state-based classification of both the loop-based AID and the FCD AID. Section 5 gives the performance results of the FCD AID on 2 Dutch highways, delving deeper into the FCD AID parameters and the impact of delay. In closure, Section 6 presents a discussion on the results of the live tests.

\section{Existing Monitoring and Management}

In this study, we focus on 2 different highways, both currently equipped with the same Automated Incident Detection (AID) system based on roadside sensors (inductive loop detectors). This current system is to be compared to the FCD version. The AID system focuses on detecting congestion and other traffic incidents as quickly as possible and warns upstream traffic by using dynamic overhead speed advice signs. This allows incoming traffic to reduce their speed and avoid rearend collisions. We first describe the AID system here.

The installed AID system monitors the highways using the loop data sensor embedded in the road every few hundred meters (typically $500 \mathrm{~m}$ ). These loop data sensors are coupled to overhead speed signs. The speed sign controller keeps a running average of the individual vehicle speeds registered by each loop data sensor. The speed $v_{\text {cur }}$ of the loop data sensor is updated when a car passes the sensor. When a car passes with 


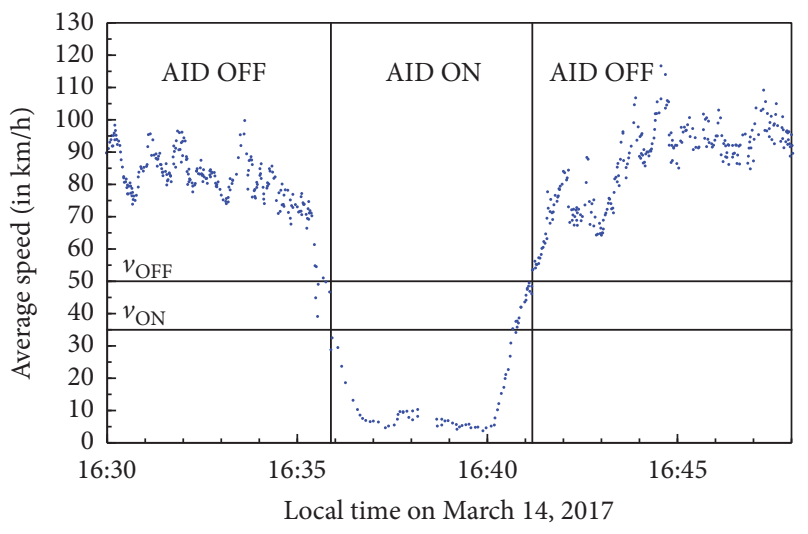

FIgure 1: AID switching. The AID switches on and off based on the average speed of the loop sensor. The blue dots show the average of all samples across all lanes. The AID goes on when the average drops beneath the $v_{\mathrm{ON}}$ threshold just before 16:36 and goes off when traffic restores after 16:41.

speed $v_{s}$ lower than $v_{\text {cur }}$, this indicates traffic slowing down and $v_{\text {cur }}$ is changed to $\left(1-\alpha_{\text {dec }}\right) \cdot v_{\text {cur }}+\alpha_{\text {dec }} \cdot v_{s}$. If $v_{s}>v_{\text {cur }}$, the update is done analogously but with a different weighting factor: $\left(1-\alpha_{\text {acc }}\right) \cdot v_{\text {cur }}+\alpha_{\text {acc }} \cdot v_{s}$. This update process aims at quickly reacting to traffic slowing down (high $\alpha_{\mathrm{dec}}=$ dropping quickly) while only gradually raising the average when the congestion is dissolving (lower $\left.\alpha_{\text {acc }}\right) \cdot \alpha_{\text {acc }}$ and $\alpha_{\text {dec }}$ are limited to the $[0,1]$ range and can be modified by road operators to tune the system performance for the specific location.

The averages calculated by the loop sensors are monitored to detect congestion incidents. An incident is reported when $v_{\text {cur }}$ of a specific location drops below a predefined threshold $v_{\mathrm{ON}}$, which triggers an AID ON message and activates the overhead sign. When traffic conditions improve and $v_{\text {cur }}$ rises above the $v_{\mathrm{OFF}}$ threshold, this triggers an AID OFF message to deactivate the sign and end the incident. The thresholds can be set differently to avoid excessive switching of the road sign. Figure 1 shows this process for a local jam on the A58.

The AID ON message triggers the speed signs of all portals within a configurable range termed look-ahead distance LAD. This trigger overrules the normal maximum allowed speed (typically $130 \mathrm{~km} / \mathrm{h}$ on the Dutch highways). While this behaviour is configured by the road operator for each specific location, for the roads in this study, the AID ON triggers a speed sign display of $50 \mathrm{~km} / \mathrm{h}$ for all speed signs within a range of $700 \mathrm{~m}$. With loop sensors and portals spaced approximately $500 \mathrm{~m}$ apart, generally 2 speed signs are activated. To avoid abrupt speed changes, a sign of $70 \mathrm{~km} / \mathrm{h}$ is also triggered upstream of the first $50 \mathrm{~km} / \mathrm{h}$ sign. To further draw attention to the speed limits changes, flashing lights accompany the speed signs when they differ from the limit upstream.

\section{FCD Extensions}

The AID system from the previous section was developed throughout the 1970s and 1980s and further tuned in the past decades. While this system has proven its efficacy over time, it is still limited by its input (loop sensor) data, only available on specific roads (about a quarter of all Dutch motorways). By implementing this system using FCD and new and/or cheaper signage systems (e.g., in-car delivery and simpler roadside signs), its application area is greatly increased.

FCD consists of individual vehicle samples generated by tracked probe vehicles. Each sample consists of a pair of coordinates, an anonymized vehicle identifier, a timestamp, vehicle heading, and a measured speed estimate $v_{s}$. The samples are matched to an underlying representation of the road network with segments of at most $50 \mathrm{~m}$. This fine granularity allows monitoring traffic variances on a detailed level without the need for extensive trip generation to determine intermediate segments between successive probes.

In the FCD system of this study, samples are generated every second by the vehicle probe and sent to the central server every $10 \mathrm{~s}$ for further processing by the AID software. As the measured samples are subject to measurement noise, the FCD system applies several filters to reduce errors. First, erroneous measurements (e.g., missing fields and inconsistent data) and irrelevant samples (e.g., outside of study area) are discarded. For each remaining sample, the nearest road segments in the underlying network are identified and filtered (taking into account vehicle heading and road type) to obtain a segment match for the sample. For new vehicles, a new trip is then generated starting from this segment while, for known vehicles, their trip is extended with the new segment. This creates/updates the vehicle trip history and helps to further reduce measurement noise as only valid trips are considered by the AID algorithm. A sample is only taken into account when the vehicle trip is guaranteed to be on the route. This eliminates, for example, vehicle samples from neighbouring roads for which the trip segment history is inconsistent (e.g., consecutive segments are not connected in the network). It also omits samples from vehicles coming from entry ramps (as they have not yet been on the highway long enough) which are undesirable as they are still accelerating.

Using the trip samples from the validated vehicles, the FCD AID is implemented analogously as the loop AID. On each segment of the monitored routes, virtual sensors are defined in software. While the loop AID has physical sensors every $500 \mathrm{~m}$ on average, the FCD AID has simulated sensors on each segment of the underlying road network, spacing sensors roughly every $50 \mathrm{~m}$. When valid vehicle probe samples are received, the current average speed $v_{\text {cur }}$ on the mapped segment is updated analogously to the loop AID system (taking into account weighting factors according to the speed of the sample, see Section 2). This update process is event-driven, with averages being calculated as soon as the sample is received.

The calculated averages are then used (as in the loop AID) to trigger AID ON/OFF messages and incident reports. Each virtual sensor will trigger a congestion notification when it goes below the lower speed threshold $v_{\mathrm{ON}}$ and a free-flow notification when the average speed restores to above the upper speed limit threshold $v_{\mathrm{OFF}}$. These notifications are monitored by virtual FCD speed signs, which are modelled on the locations where the physical loops are installed along the route. Each virtual FCD speed sign monitors the virtual FCD 


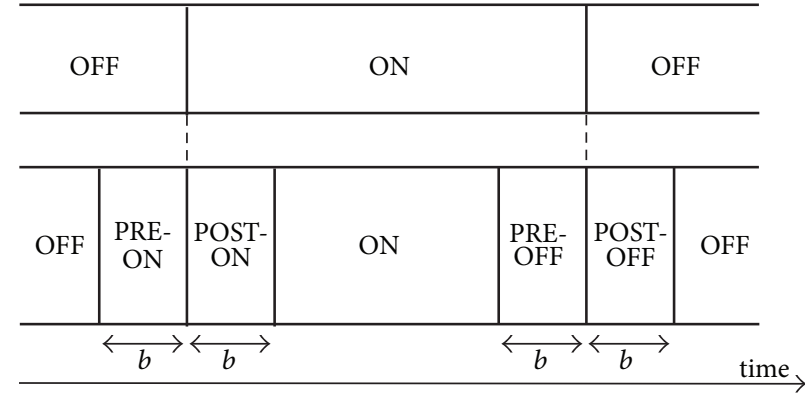

(a)

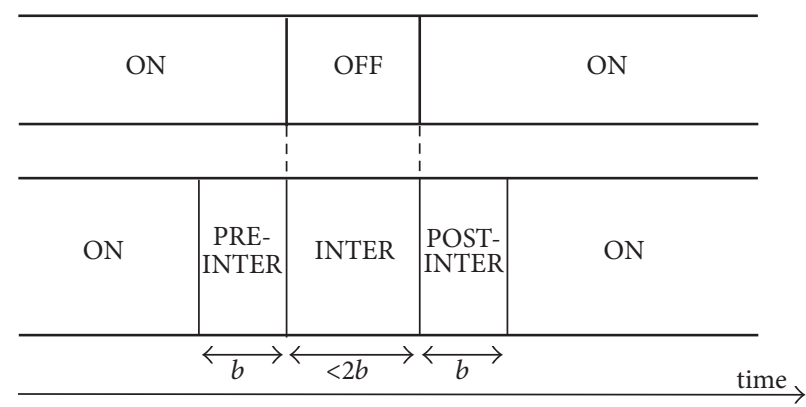

(b)

Figure 2: State classification. The loop benchmark states are converted to a ground truth classification. (a) denotes the buffering near normal $\mathrm{ON} / \mathrm{OFF}$ switches; (b) shows what happens when the loop switches $\mathrm{ON} \rightarrow \mathrm{OFF} \rightarrow \mathrm{ON}$ in a short time window.

sensors in a configurable downstream range defined as the look-ahead distance LAD, typically $500-1000 \mathrm{~m}$. As the virtual sensors are only $50 \mathrm{~m}$ apart, each individual virtual speed sign is influenced by multiple virtual sensors, typically $10-20$ assuming segments of $50 \mathrm{~m}$. This fine granularity allows for finer signage and more smooth control. The virtual speed sign will trigger an AID ON message when one of its associated segments detects congestion. Note that congestion will be detected when $v_{\text {cur }}$ drops below the $v_{\mathrm{ON}}$ threshold. Due to $\alpha_{\mathrm{dec}}$ smoothing the $v_{\text {cur }}$ decline, this requires multiple samples, effectively ensuring that a single erroneous sample cannot trigger a congestion event. When congestion dissipates, the AID OFF message is sent when all of the associated congested virtual sensors have reported free-flow again, similar to the loop AID. The created AID messages are sent in real-time to the local highway operator to be shown on the physical sign infrastructure. Note that the preceding $70 \mathrm{~km} / \mathrm{h}$ advice of the loop AID is also replicated here but it is not taken into account for this analysis.

\section{Evaluation Methodology}

To compare both AID systems and investigate the effect of FCD system delay, the FCD AID is benchmarked against a ground truth constructed out of the loop AID. While the FCD AID inherently covers the entire road network and allows for signage on every road (segment), the evaluation here is limited to the locations equipped with loop sensors. This favours the loop AID as their performance is best at those locations (in contrast to unmonitored locations in between loops or on unequipped highways). Additionally, as the loop AID (with its shortcomings) is taken as the ground truth, the FCD AID is limited to mimicking and penalized for each difference, thereby limiting possible improvements. However, the loop AID is the best automated benchmark available, with realistic traffic conditions free of model assumptions.

The aim of the analysis is to determine if the input FCD AID is able to match the performance of the loop AID. Delay is considered the most important issue here, as alerting drivers need to be done as soon as possible. The loop AID has little delay as the data measurements (= individual vehicles passing a loop sensor) are directly coupled to the sign controller at the road location itself. The FCD however requires several steps, introducing delay. First of all, it is dependent on the vehicle polling frequency. While vehicles measure their location at fixed intervals, transmitting the data is done in batches (grouping several GPS samples in one communication). This lowers the battery usage of the vehicle probes but adds buffering delay. If a vehicle would only transmit its data once every minute, this would introduce an average delay of $30 \mathrm{~s}$. Next, communicating the data to the central sign controller over the public communication network also introduces a transmission delay of several seconds. Additionally, as only a small subset of all vehicles is sampled, extra sampling delay is accrued as the FCD AID needs to wait for a monitored vehicle to pass the location under study. The time between samples can vary significantly; for example, if there is very little traffic at night, hardly any samples will be available. In general, the delay is coupled to the penetration rate (as twice as many probes roughly halve the sampling delay). In our study, the delay was in the order of a couple of seconds to a few minutes. Finally, processing the data of a country-wide network requires some time (to filter out the relevant messages from the erroneous or irrelevant messages, mapping samples to road segments), adding processing delay (in the order of seconds) before being able to transmit instructions to AID sign. The main focus of this paper is to determine if the FCD AID can overcome these delays.

To get a clear view on the FCD AID behaviour at AID change times for a single location, we construct a ground truth out of the benchmark AID consisting of several states. By calculating the time spent by the FCD AID in each of the different states, we can derive high level behaviour of the AID.

Throughout the evaluation study period, the loop AID switches ON and OFF. To zoom in on the behaviour at switching times, we set a predefined buffer $b$ (of, e.g., 60 s) around each state change. When the loop AID switches from OFF to ON at time $t_{1}$, the period $\left[t_{1}-b, t_{1}\right]$ is the PRE-ON state. Analogously, the period $\left[t_{1}, t_{1}+b\right]$ is the POST-ON. The period $\left[t_{1}+b, t_{2}-b\right]$ is defined as the ON state (with $t_{2}$ being the time at which the AID would switch back OFF), corresponding to a state when the AID should clearly be on. This classification is shown in Figure 2(a). When the FCD AID would switch on too early, it would spend time in the PRE-ON period. Analogously, we can define the PRE-OFF 


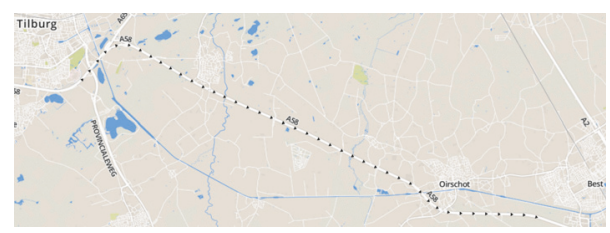

Figure 3: A58 test route running from Tilburg to Eindhoven, covering $19 \mathrm{~km}$.

and POST-OFF states at the end of the loop AID event $\left(t_{2}\right)$ and OFF for all time instants not near an AID event. With the state differentiation between PRE-ON and OFF, it is clear to see when the FCD AID is more proactive in warning (by switching on earlier) instead of just wrong.

When AID changes occur rapidly, it is hard to judge if the AID should be on or off, given that the one system might still be on for the first event while the other is already on for the next event. With an imperfect benchmark, this is even harder. To mediate this problem, we group individual loop AID ON periods if they are close together in time. If 2 events are within $2 \cdot b$, they are considered to be 1 big event. The time in between consists of an INTER state. The period before and after the gap $(b s)$ is defined as PRE-INTER and POST-INTER. This mechanism is shown in Figure 2(b).

This classification yields 9 states covering the entire day/study period. The AID system under study can now be evaluated by calculating the time spent in every state, taking into account the loop AID result. This is done in Tables 2 and 3 . When the loop AID system under study would be compared to itself, it would always score maximal for the ON or OFF state and 0 for the other. The total time per column is fixed for all evaluated AID systems. The FCD AID (and potential others) would differ from the loop AID by having nonzero values at the zero-positions (e.g., $10 \%$ of the max) and having nonmaximal values at the other positions (e.g., 90\%). These denote false positives (= indicating ON during the OFF state) and false negatives (= indicating OFF during $\mathrm{ON}$ ) but also soft false positives (e.g., showing ON during PRE-ON and POST-OFF) and soft false negatives (e.g., showing OFF during POST-ON and PRE-OFF). The raw figures themselves indicate the time per state and can be used to characterize the system.

\section{Results}

5.1. Experimental Setup. To study the FCD AID system, it was deployed on 2 different highway stretches in the Netherlands. The first test route, shown in Figure 3, is the eastbound A58 running from Tilburg to Eindhoven between highway kilometre markers 35 and 16, spanning $19 \mathrm{~km}$. On this route, 33 locations are equipped with loop sensors and AID overhead signs on which the evaluation will be focused. The route itself typically has a morning and evening rush hour peak during which traffic jams arise (at highway on-/offramps) that propagate upstream along the highway.

The second route, shown in Figure 4, is the southbound A27 running from Utrecht to Gorinchem between highway

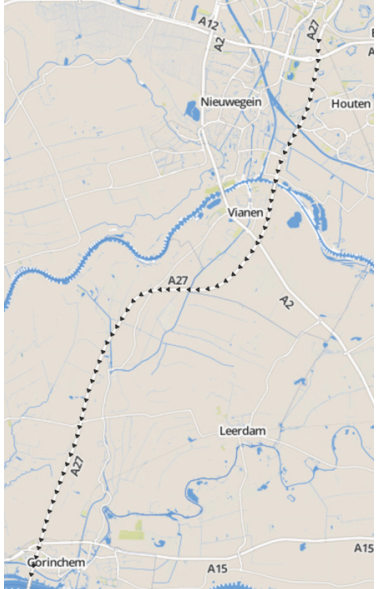

FIGURE 4: A27 test route, running from Utrecht (north) to Gorinchem (south), covering $37 \mathrm{~km}$.

TABLE 1: Experimental FCD AID configurations deployed on the test routes.

\begin{tabular}{lccccc}
\hline CONFIG & $\alpha_{\mathrm{acc}}$ & $\alpha_{\mathrm{dec}}$ & $\begin{array}{c}v_{\mathrm{ON}} \\
(\mathrm{km} / \mathrm{h})\end{array}$ & $\begin{array}{c}v_{\mathrm{OFF}} \\
(\mathrm{km} / \mathrm{h})\end{array}$ & $\begin{array}{c}\mathrm{LAD} \\
(\mathrm{m})\end{array}$ \\
\hline$c_{1}$ & 0.4 & 0.3 & 35 & 45 & 750 \\
$c_{2}$ & 0.4 & 0.4 & 35 & 45 & 750 \\
$c_{3}$ & 0.4 & 0.5 & 35 & 45 & 750 \\
$c_{4}$ & 0.4 & 0.5 & 35 & 45 & 900 \\
$c_{5}$ & 0.4 & 0.3 & 35 & 45 & 1250 \\
$c_{6}$ & 0.4 & 0.4 & 35 & 45 & 1250 \\
$c_{7}$ & 0.4 & 0.5 & 35 & 45 & 1250 \\
$c_{8}$ & 0.4 & 0.5 & 35 & 45 & 1400 \\
\hline
\end{tabular}

kilometre markers 72 and 35 (spanning $37 \mathrm{~km}$ ) with 61 AID equipped measurement locations.

The FCD used in this experiment was obtained from a commercial company monitoring the entire Dutch road network. The FCD penetration rate was determined for the A58 by counting all vehicles on an individual segment of both the FCD and the loop detectors between March 13 and March 17 resulting in an average weekday coverage of $5.93 \%$. As mentioned above, samples were logged on device every second but only transmitted to the server every $10 \mathrm{~s}$. Comparing the timestamp of the samples to the received timestamp, an average $2 \mathrm{~s}$ communication delay was found before the AID software received the data. The AID calculation was implemented in Go, mapping probe samples to road segments, maintaining speed averages and creating AID messages, adding on average another $2 \mathrm{~s}$ processing delay. After AID calculation, the results are transmitted to the Dutch roadside traffic operators.

To examine the effects of the individual AID parameters, 8 different configurations (see Table 1) were selected and deployed in parallel using the live FCD system. $\alpha_{\mathrm{acc}}$ and speed thresholds were kept constant as initial experiments indicated good correspondence to the benchmark. Having different 


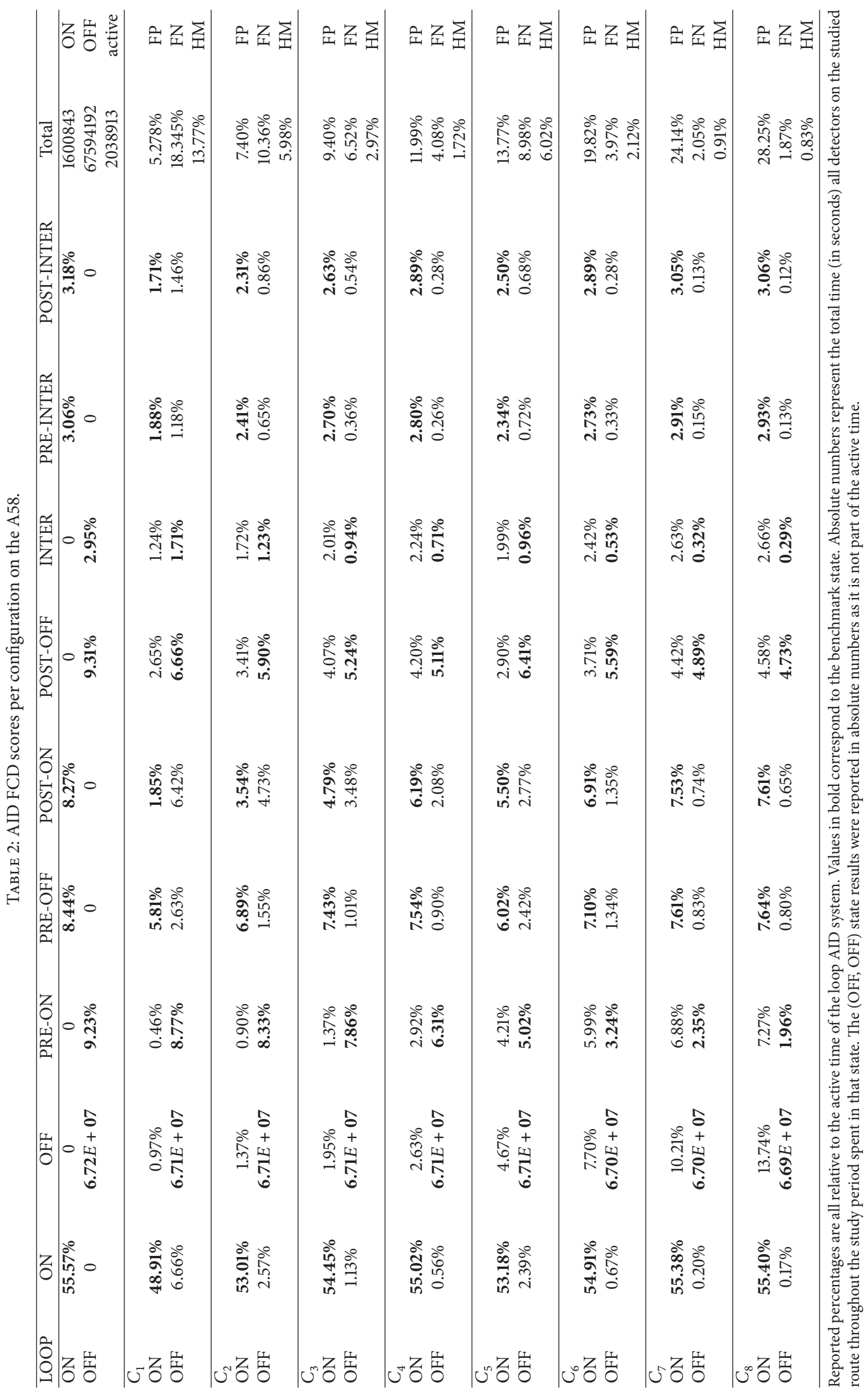




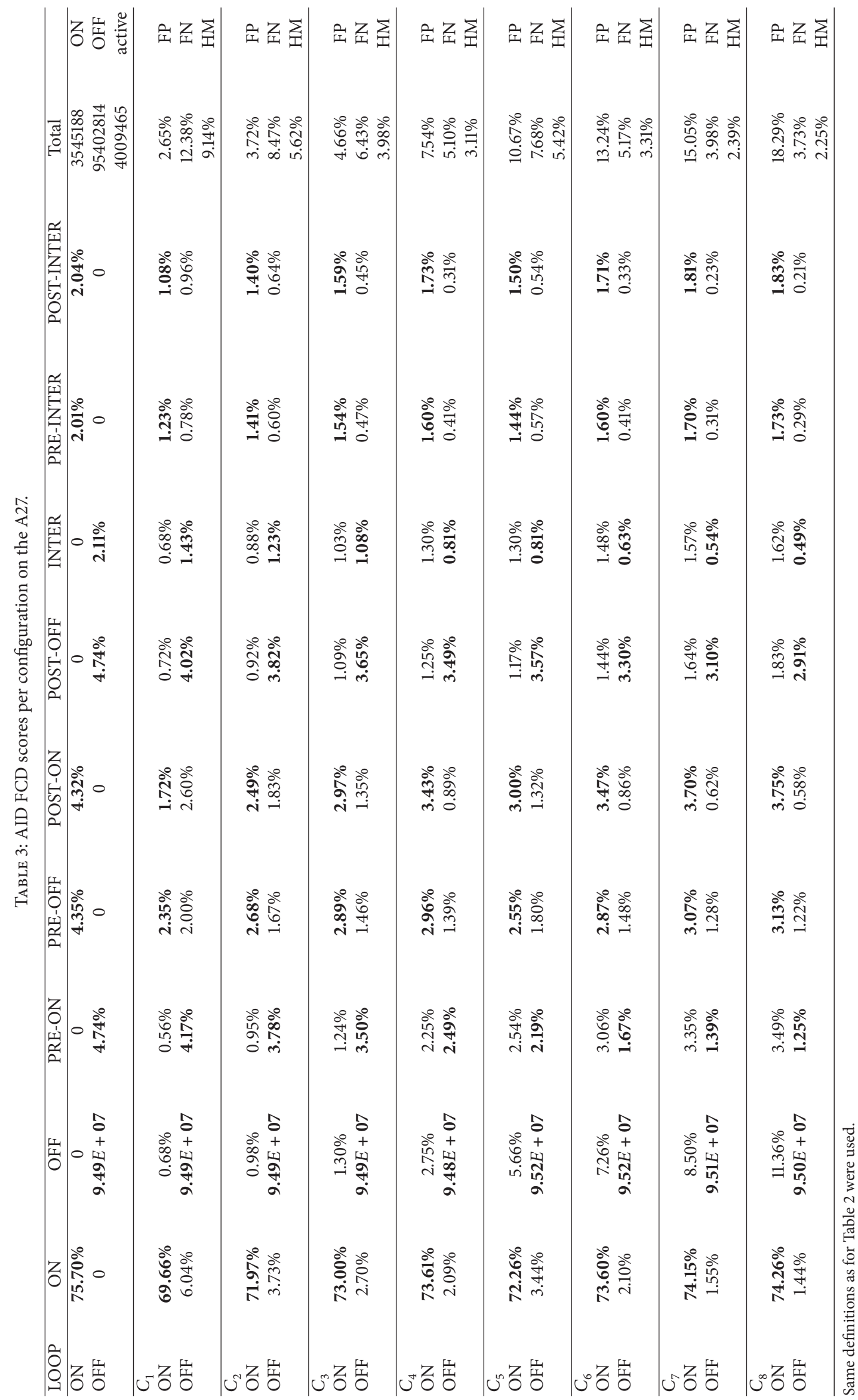


speed thresholds as the ground truth resulted in large discrepancies when traffic conditions evolved slowly. The look-ahead distance LAD and $\alpha_{\mathrm{dec}}$ were varied to investigate more rapid $\mathrm{ON}$-switching. Note that the main intent in this paper is to investigate the feasibility of the FCD AID system. Deriving optimal parameter configurations was not focused on as these values vary depending on the desired switching behaviour by the road operator. Furthermore, as with loop AID, the parameters can be varied along the route, depending on local road properties. To derive optimal (local) parameter configurations, many more configurations should be run, but this is out of scope for the limited system setup. Also note that the loop AID benchmark should not be too strictly followed as it has its own disadvantages (see the discussion section of this work).

5.2. FCD AID Configuration Results. The different FCD AID setups were compared to the installed loop-based AID logs provided by the Dutch highway operator for 4 weeks (1-28 March 2017, excluding 6 and 7 March for maintenance). The loop-based AID logs were categorized as described in Section 4 and used as the ground truth for the AID FCD. Note that the automated installed AID system can be manually overruled by roadside operators. These overrides were identified and removed from the analysis as we are only interested in the automated operation of the system. Tables 2 and 3 show the overall performance of the different setups. All percentages are relative to the total time the loop-based system is active (= everything except the OFF period state). Excluding the OFF periods (typically during the night) gives a clearer picture of the relevant active periods. The false positive percentages FP were calculated as the ratio of the time the FCD system triggers ON while the loop-based system shows OFF to the active time. The false negative percentage FN denotes the percentage of active time the FCD AID is OFF while the loop AID is ON. Additionally, the "hard misses," $\mathrm{HM}$, are also reported, denoting the percentage of time the loop-based AID is ON while the FCD AID is OFF and will stay OFF for more than $60 \mathrm{~s}$. This represents the instances in which the FCD AID misses a traffic jam reported by the loopbased system. Figures 5 and 6 show the trade-off between the false positives and the false negatives, along with the hard misses.

With these numbers, the difference in traffic conditions between both test routes can be quantified. While the A27 is almost twice as long as the A58 route, it is also more impacted by traffic jams. On average, a loop AID sign on the A27 was active $3.58 \%$ of each day (3095 s) while an A58 loop AID sign was only active $2.31 \%$ (1999s). While the A27 has several intersections (with other highways) causing turbulence and congestion near on- and off-ramps, the A58 is less congested, largely consisting of backwards propagating traffic jams. As indicated by the hard miss percentages, FCD AID performs slightly better for the A58 with a more predictable traffic pattern than for the $\mathrm{A} 27$, with its more dynamic traffic. Looking at configuration $\mathrm{C}_{4}$, the hard miss percentages for the A58 can be reduced to below 2\%, while most configurations for the A27 stay above 3\%. The false negative percentages of $4.08 \%$ and $5.10 \%$, respectively, for the

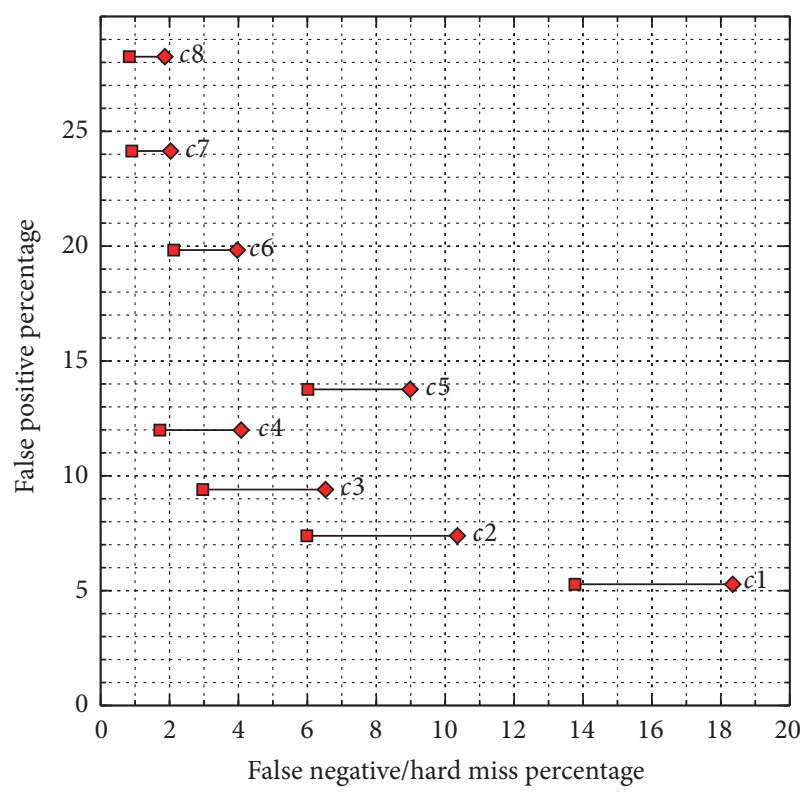

FIGURE 5: Trade-off between false positives and false negatives on the A58. Diamonds indicate the false negative percentages while the squares denote the hard miss percentages.

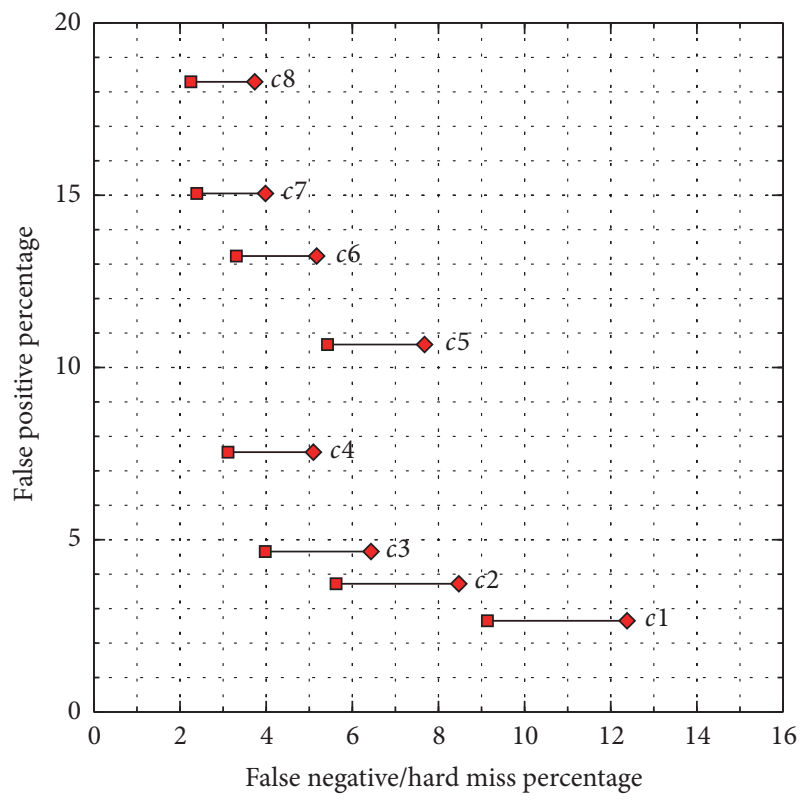

FIGURE 6: Trade-off between false positives and false negatives on the A27. Diamonds indicate the false negative percentages while the squares denote the hard miss percentages.

A58 and A27 roughly translate to a period of 82 and 158 seconds per day, respectively, when an FCD AID overhead sign is not showing while the loop AID would be showing.

Overall, the figures show the typical trade-off between triggering more (having more false positives and less false negatives) and triggering less (resulting in less false positives and more false negatives). As shown by the plotted hard 
misses, on average $46 \%$ of the false negatives on the A58 are soft misses (35\% on the A27), resulting from the FCD AID switching on later than the loop AID. To counter this delay and obtain the desired recall rate (as deemed necessary by the road operator), the configuration parameters can be tuned. Increasing the look-ahead distance results in more virtual FCD AID loop sensors being taken into account and therefore more AID ON triggers. While the loop AID has an average interloop distance of $700 \mathrm{~m}$, the look-ahead distances in the experimental setup are higher, as the FCD AID needs to counter the inherent delays (predominantly sampling and buffering delay). By having a higher LAD, backwards propagating traffic jams are more adequately detected. Having a higher $\alpha_{\text {dec }}$ also reduces false negative rates, as low speed samples impact the average speed more, reaching the lower threshold quicker. Having too high $\alpha_{\mathrm{dec}}$ however leads to overweighting of individual samples, attributing too much importance to individual probe vehicles.

5.3. Full State Comparison. While the desired trade-off is dependent on the intended use case, configuration $c_{4}$ is chosen here to further investigate, corresponding to a use case where both false positives and false negatives are to be minimized, but with a higher focus on false negatives (for safety). Table 4 shows a complete comparison between the loop AID and FCD AID on the A58 with the FCD AID also being categorized in 9 states as done for the loop AID. Table 5 shows the same for the A27 test case. This breakdown better displays the different contributing factors to the false negative and false positive percentages. The nondiagonal numbers indicate the mismatch between both systems. Of the total reported $4.08 \% \mathrm{FN}$ for the $\mathrm{A} 58$ setup, about $1 / 3$ (1.23\%) originates from the (POST-ON, PRE-ON) state, indicating the FCD AID switching on too late. Interestingly, for the total $11.99 \%$ false positive rate, the major contributions are from the (POSTOFF, PRE-OFF) state $(2.66 \%)$ corresponding to the FCD switching off too late and the (PRE-ON, POST-ON) state (1.83\%) corresponding to the FCD AID switching on earlier than the loop AID. For the A27 setup, the biggest contribution to the false negative rate comes from the (PRE-OFF, POSTOFF) state $(0.84 \%)$, with FCD AID thus switching off sooner.

Looking at the column/row totals for the 3 INTER states in both AID systems, FCD AID spends 91571 s in all "inter" states on the A58 while it spends $273597 \mathrm{~s}$ on the A27. Similarly, the loop AID spends $187268 \mathrm{~s}$ for the A58 and 245707 s on the A27. These numbers again show the difference between both routes. Taking into account the fact that the A27 covers nearly twice as many connectors as the A58 but only reports $31 \%$ more time, the A58 has more "inter" time per AID sign compared to the A27. This is due to the systematic backwards propagating traffic jams resulting in stop-and-go traffic and the associated AID INTER switching. FCD AID only spends half the time the loop AID does for the A58 as with only a limited subset of vehicles; it smooths out these patterns, yielding less frequent switching. For the A27, the FCD AID spends roughly the same amount of time in the "inter" states as the loop AID.

\section{Discussion}

In the previous sections, a state classification methodology was presented along with the results of a 4-week field test in which different FCD AID system configurations were deployed using data from a country-wide FCD system monitoring $5.93 \%$ of all traffic present on 2 routes with different traffic and congestion properties. Results were successfully coupled back to the roadside operators, proving full system technical feasibility. To score the FCD AID, it was compared to the installed loop AID and false negative percentages of 4-5\% (of the total active benchmark AID) were obtained for a selected FCD AID configuration. This translates to a false negative of 82 and $158 \mathrm{~s}$ per AID sign per day on the A58 and A27 route, respectively. Before considering the applicability, this number needs to be further put into context.

First, note that the loop AID is considered to be a golden standard, with its performance validated and tuned over the past decades. However, it also has its known disadvantages, for example, its limited spatial resolution. The benchmark relies on local loop measurements but is blind for traffic problems/congestion forming between successive loop locations on the same route while FCD systems can operate on every part of the route. The evaluation itself was limited to the locations with loop equipment, with loop AID signage between locations by default being that of the upstream AID sign. The AID for those intermediate locations inherently suffers a delay (which the FCD AID does not have) but is not considered here. Due to the golden standard, instances where FCD would warn earlier than loops are also counted as false positives, limiting FCD performance to just mimicking the loop AID and not allowing improvements. Secondly, the FCD AID presented here was not tuned to specific locations (e.g., ramps and intersections) but used the same logic on every part of the route. This was done to better investigate the potential of a country-wide system, for which initial manual tuning for each road would be cumbersome. However, as seen by comparing the test cases, different traffic situations warrant different configurations. For the installed loop AID, each location is also tunable in its configuration. Thirdly, the results were generated on a pilot AID system with a limited sample of live traffic while the loop system monitors all vehicles and is directly coupled to the AID signage. This results in the FCD AID being currently disadvantaged as it could perform even better with dedicated infrastructure designed to optimize delay. Lastly, the deployed FCD AID was made to mimic the current operational algorithm (with weighted averages, upper and lower thresholds, ...) to allow best integration with the existing systems. More advanced methods with forecasting models or flow optimization can ultimately be used.

Given these disadvantages, the FCD AID results do indicate that FCD presents a valid source of traffic data for live traffic management systems. For the studied period, road setup, and coverage, the study here shows that a live AID system can be deployed with reasonable differences. While it is tempting to put an exact number on the performance of the system, it is practically void as a "perfect" AID system cannot be defined, as trade-offs will always need to be made. 


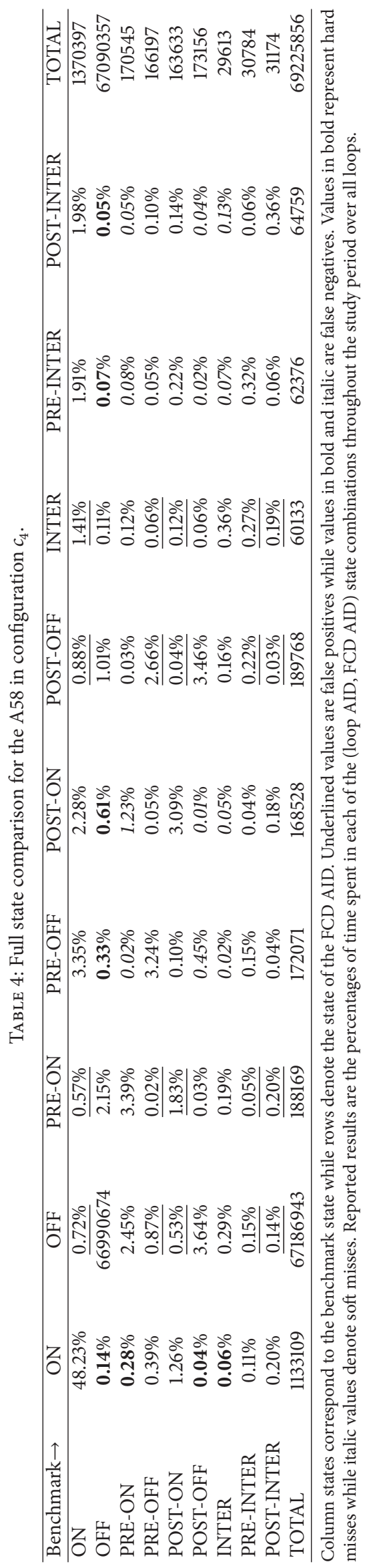




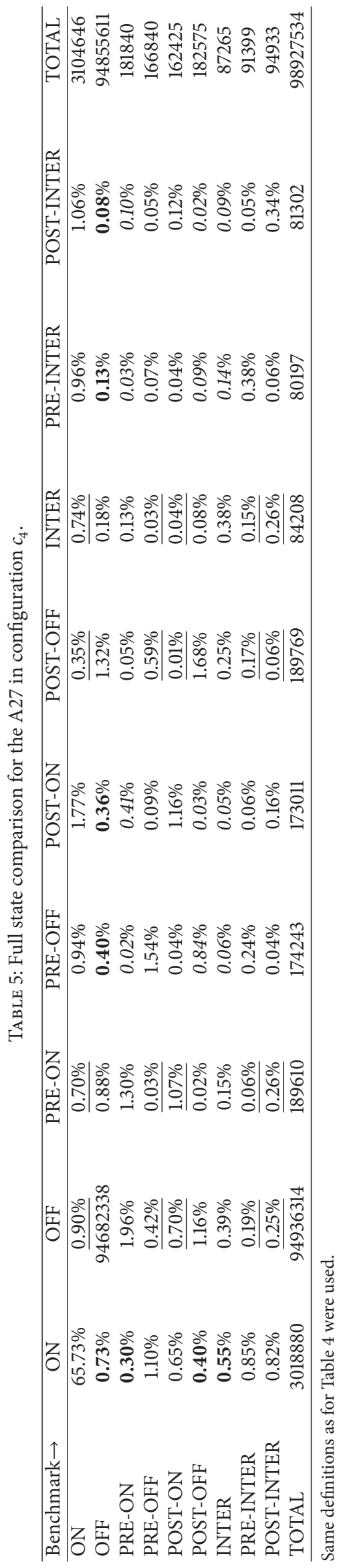


It is however clear that the presented AID system presents a cost efficient alternative or addition to loop AID, as data fusion techniques can only improve the information for the loop AID. For areas currently without loop equipment, FCD AID offers a quick and practical solution. By leveraging FCD technology in these areas, we can reduce traffic congestion, combat pollution, and increase safety.

\section{Disclosure}

Maarten Houbraken was a Ph.D. fellow of the Research Foundation-Flanders (FWO-Vlaanderen).

\section{Conflicts of Interest}

The authors declare that there are no conflicts of interest regarding the publication of this paper.

\section{Acknowledgments}

The authors would further like to acknowledge the Dutch roadside operator, Rijkswaterstaat (RWS), for providing the benchmark data. The authors would also like to acknowledge RWS and the rest of the project partners for the fruitful discussions.

\section{References}

[1] B. Coifman, "Estimating travel times and vehicle trajectories on freeways using dual loop detectors," Transportation Research Part A: Policy and Practice, vol. 36, no. 4, pp. 351-364, 2002.

[2] H. X. Liu, X. He, and W. Recker, "Estimation of the timedependency of values of travel time and its reliability from loop detector data," Transportation Research Part B: Methodological, vol. 41, no. 4, pp. 448-461, 2007.

[3] C. Chen, K. Petty, A. Skabardonis, P. Varaiya, and Z. Jia, "Freeway performance measurement system: mining loop detector data," Transportation Research Record: Journal of the Transportation Research Board, vol. 1748, pp. 96-102, 2001.

[4] B. Z. Yao, P. Hu, M. H. Zhang, and M. Q. Jin, "A support vector machine with the tabu search algorithm for freeway incident detection," International Journal of Applied Mathematics and Computer Science, vol. 24, no. 2, pp. 397-404, 2014.

[5] J. Xiao, X. Gao, Q.-J. Kong, and Y. Liu, "More robust and better: a multiple kernel support vector machine ensemble approach for traffic incident detection," Journal of Advanced Transportation, vol. 48, no. 7, pp. 858-875, 2014.

[6] C. Oh, S. Park, and S. G. Ritchie, "A method for identifying rear-end collision risks using inductive loop detectors," Accident Analysis \& Prevention, vol. 38, no. 2, pp. 295-301, 2006.

[7] U. S. D. of Transportation, "DOT ITS Knowledge Resources," 2017, http://www.itscosts.its.dot.gov/.

[8] J. Li, H. van Zuylen, and G. Wei, "Diagnosing and interpolating loop detector data errors with probe vehicle data," Transportation Research Record: Journal of the Transportation Research Board, vol. 2423, pp. 61-67, 2014.

[9] S. Cohen and Z. Christoforou, "Travel time estimation between loop detectors and Fcd: a compatibility study on the Lille Network, France," Transportation Research Procedia, vol. 10, pp. 245-255, 2015.
[10] A. D. Patire, M. Wright, B. Prodhomme, and A. M. Bayen, "How much GPS data do we need?" Transportation Research Part C: Emerging Technologies, vol. 58, pp. 325-342, 2015.

[11] M. Vanlommel, M. Houbraken, P. Audenaert, S. Logghe, M. Pickavet, and P. De Maeyer, "An evaluation of section control based on floating car data," Transportation Research Part C: Emerging Technologies, vol. 58, pp. 617-627, 2015.

[12] R. Neuhold, M. Haberl, M. Fellendorf et al., "Generating a lanespecific transportation network based on floating-car data," Advances in Intelligent Systems and Computing, vol. 484, pp. 1025-1037, 2017.

[13] C.-S. Chou and A. P. Nichols, "Deriving a surrogate safety measure for freeway incidents based on predicted end-of-queue properties," IET Intelligent Transport Systems, vol. 9, no. 1, pp. 22-29, 2015.

[14] G. Klunder, H. Taale, and S. Hoogendoorn, "The impact of loop detector distance and floating car data penetration rate on queue tail warning," in Proceedings of the 3rd International Conference on Models and Technologies for Intelligent Transport Systems, 2013.

[15] K. Liu, M. Cui, P. Cao, J. Wang, and T. Tang, "Iterative bayesian estimation of travel times on urban arterials: fusing loop detector and probe vehicle data," PLOS ONE, vol. 11, no. 6, article e0158123, 2016.

[16] Y. Yuan, H. Van Lint, F. Van Wageningen-Kessels, and S. Hoogendoorn, "Network-wide traffic state estimation using loop detector and floating car data," Journal of Intelligent Transportation Systems: Technology, Planning, and Operations, vol. 18, no. 1, pp. 41-50, 2014.

[17] M. Houbraken, P. Audenaert, D. Colle et al., "Real-time traffic monitoring by fusing floating car data with stationary detector data," in Proceedings of the International Conference on Models and Technologies for Intelligent Transportation Systems, MT-ITS, pp. 127-131, Budapest, Hungary, June 2015.

[18] N.-E. El Faouzi, H. Leung, and A. Kurian, "Data fusion in intelligent transportation systems: progress and challenges-a survey," Information Fusion, vol. 12, no. 1, pp. 4-10, 2011.

[19] Y. Asakura, T. Kusakabe, L. X. Nguyen, and T. Ushiki, "Incident detection methods using probe vehicles with on-board GPS equipment," Transportation Research Part C: Emerging Technologies, 2016.

[20] E. D'Andrea and F. Marcelloni, "Detection of traffic congestion and incidents from GPS trace analysis," Expert Systems with Applications, vol. 73, pp. 43-56, 2017.

[21] R. Wang, D. B. Work, and R. Sowers, "Multiple model particle filter for traffic estimation and incident detection," IEEE Transactions on Intelligent Transportation Systems, vol. 17, no. 12, pp. 3461-3470, 2016.

[22] J. C. Herrera, D. B. Work, R. Herring, X. Ban, Q. Jacobson, and A. M. Bayen, "Evaluation of traffic data obtained via GPSenabled mobile phones: the mobile century field experiment," Transportation Research, Part C: Emerging Technologies, vol. 18, no. 4, pp. 568-583, 2010.

[23] E. Jenelius and H. N. Koutsopoulos, "Travel time estimation for urban road networks using low frequency probe vehicle data," Transportation Research Part B: Methodological, vol. 53, pp. 6481, 2013.

[24] M. Rahmani, E. Jenelius, and H. N. Koutsopoulos, "Nonparametric estimation of route travel time distributions from low-frequency floating car data," Transportation Research Part C: Emerging Technologies, vol. 58, pp. 343-362, 2015. 
[25] R.-P. Schäfer, K.-U. Thiessenhusen, and P. Wagner, "A traffic information system by means of real-time floating-car data," in ITS World Congress, pp. 1-8, 2002.

[26] A. Kinoshita, A. Takasu, and J. Adachi, "Real-time traffic incident detection using a probabilistic topic model," Information Systems, vol. 54, pp. 169-188, 2015. 


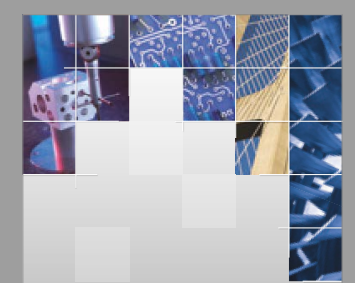

\section{Enfincering}
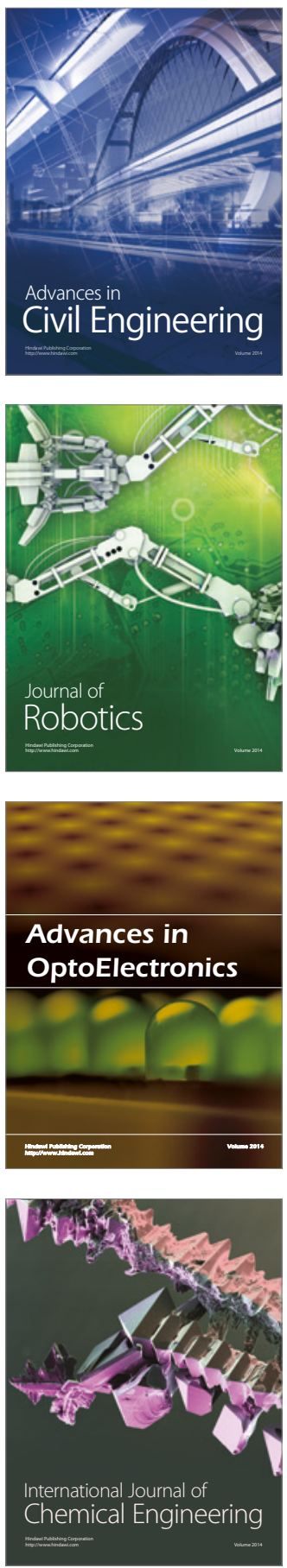

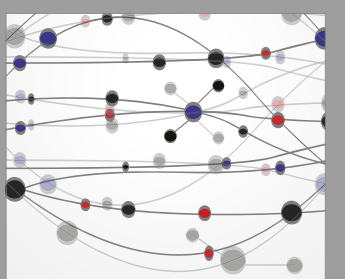

The Scientific World Journal

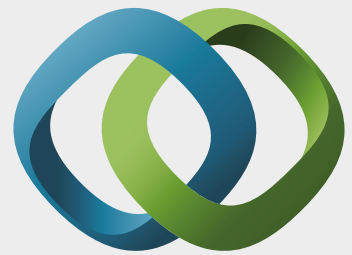

\section{Hindawi}

Submit your manuscripts at

https://www.hindawi.com
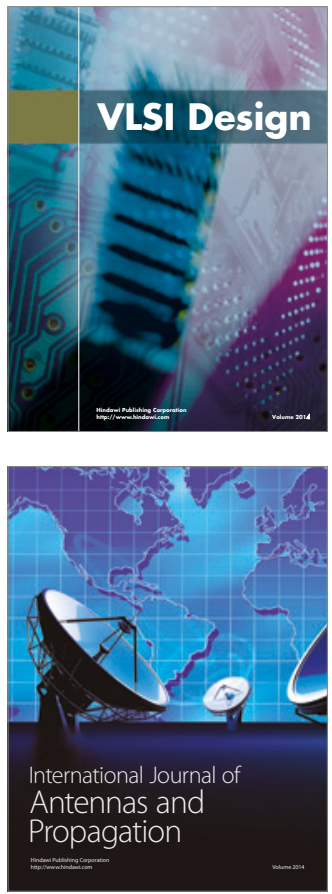

\section{Rotating}

Machinery
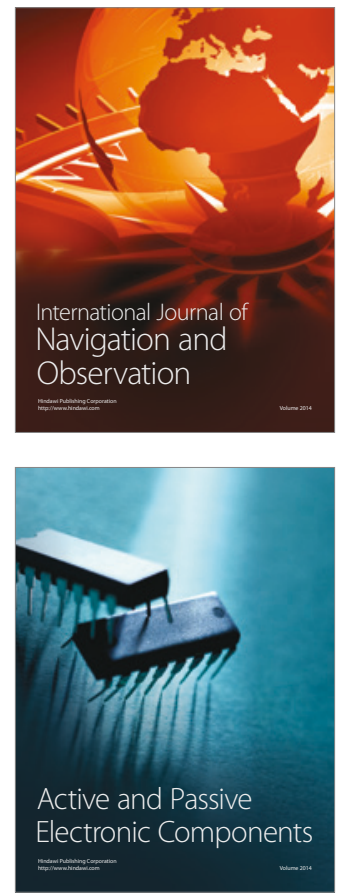
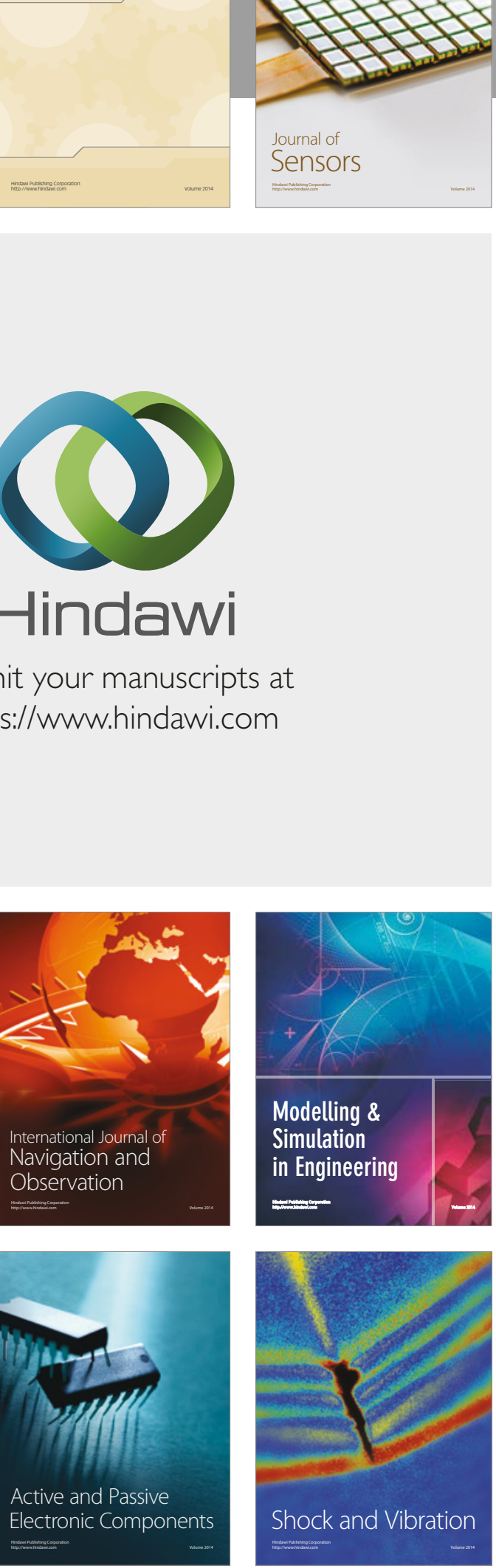
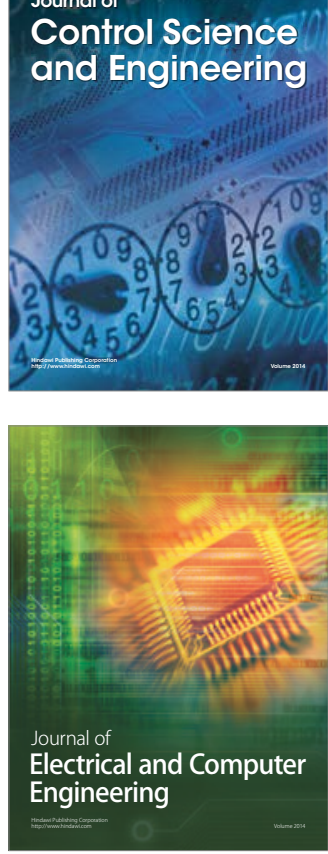

Distributed

Journal of

Control Science

and Engineering
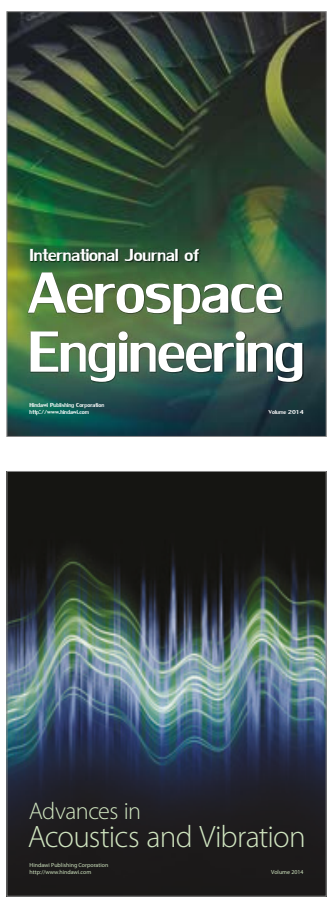

Sensor Networks 